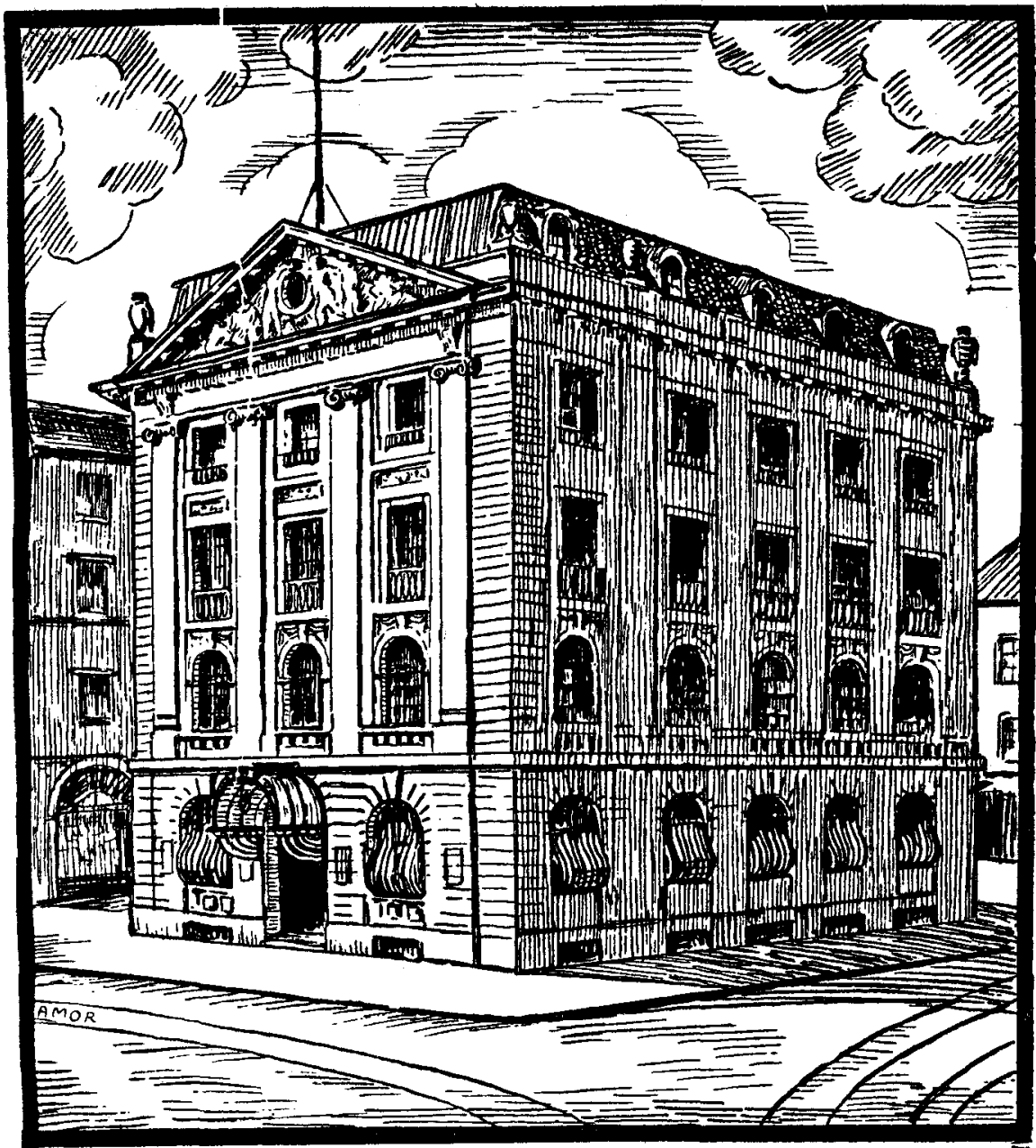

Société de

\title{
BANQUE SUISSE
}

Fondée en 1872

Capital-actions et réserves : 182 millions

$$
\text { GENEVE }
$$




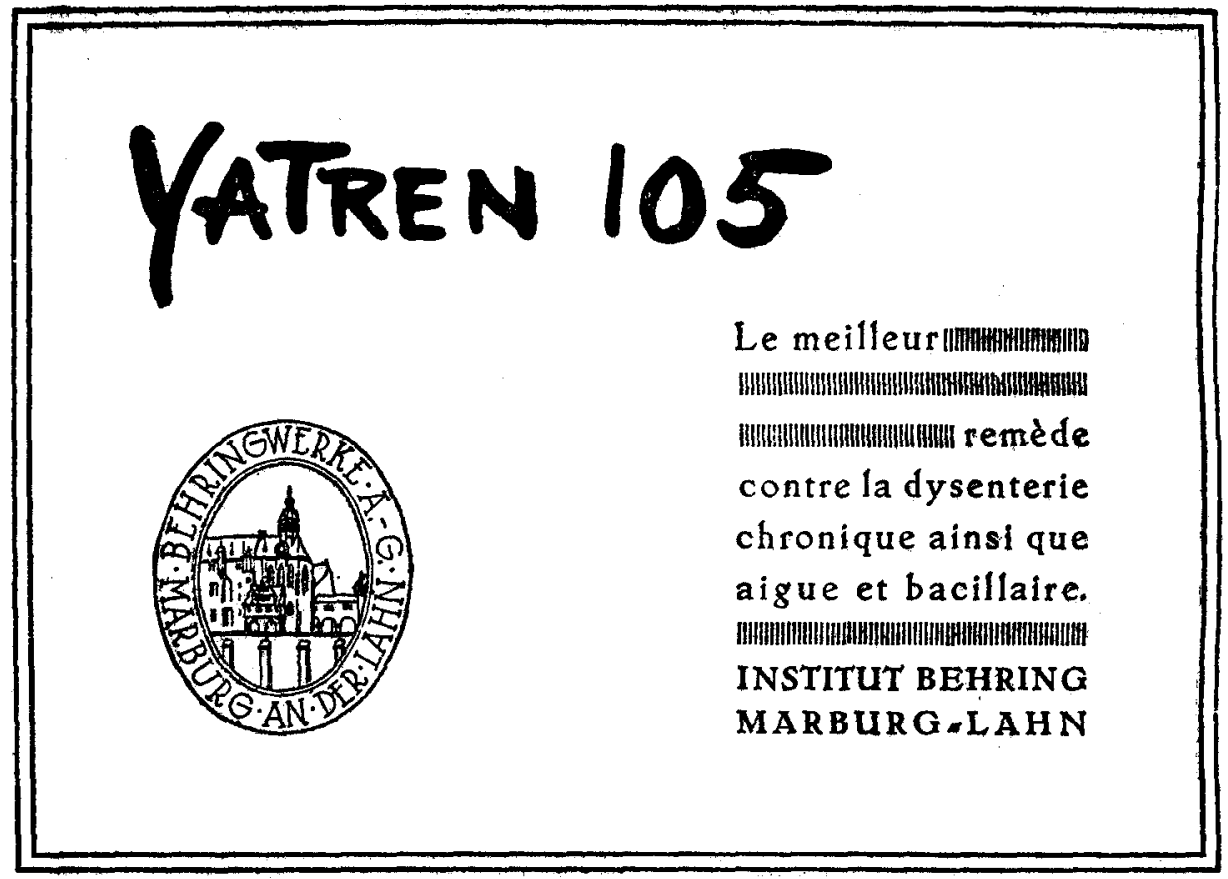

\section{REVUE INTERNATIONALE DE L'ENFANT}

31, quai du Mont-Blanc, GENĖVE

Le numéro : $1 \mathrm{fr}$. suisse. 12 numéros (un an) : $10 \mathrm{fr}$. suisses

Sommaire du numéro d'avril 1929 :

Clemens Pirquet, von Dr. Edmund Nobel, Professor an der Universität Wien.

La protection de l'enfant et le droit, par G. H. Honing, Directeur en chef de l'Association "Secours aux Sans-Logis", à Amsterdam.

The Delinquent Child in Court, by E. Hvidberg, Chairman of the Council of Guardians in Copenhagen, Denmark.

L'inspection médicale des écoles, par le $D^{r}$ L. Dufestel, Secrétaire général de la Société des médecins-inspecteurs des Ecoles de la Ville de Paris et de la Seine.

Chronique. - Nouvelles de l'U.I.S.E. - Bibliographie. 


\section{Banque Ottomane}

Capltal Lstg. 10.000.000.- dont moltié versée.

Siège central à Constantinople, GALATA.

Agences à Péra et Stamboul, ainsi que dans les principales localités de la Turquie, l'Egypte, Chypre, la Palestine, la Mésopotamie, la Perse et la Grèce.

a Londres : 26, Throgmorton Street, E.C.2.,

d Manchester : $56 / 60$, Cross Street, à Paris : 7, rue Meyerbeer ( $9^{\circ}$ Arr.). a Marseille : 41 et 43, rue Grignan, à Tunis : 68, Avenue Jules Ferry.

Se charge de toutes opérations de Banque.

Filiale pour la Syrie : Banque de Syrie et du Grand Liban.
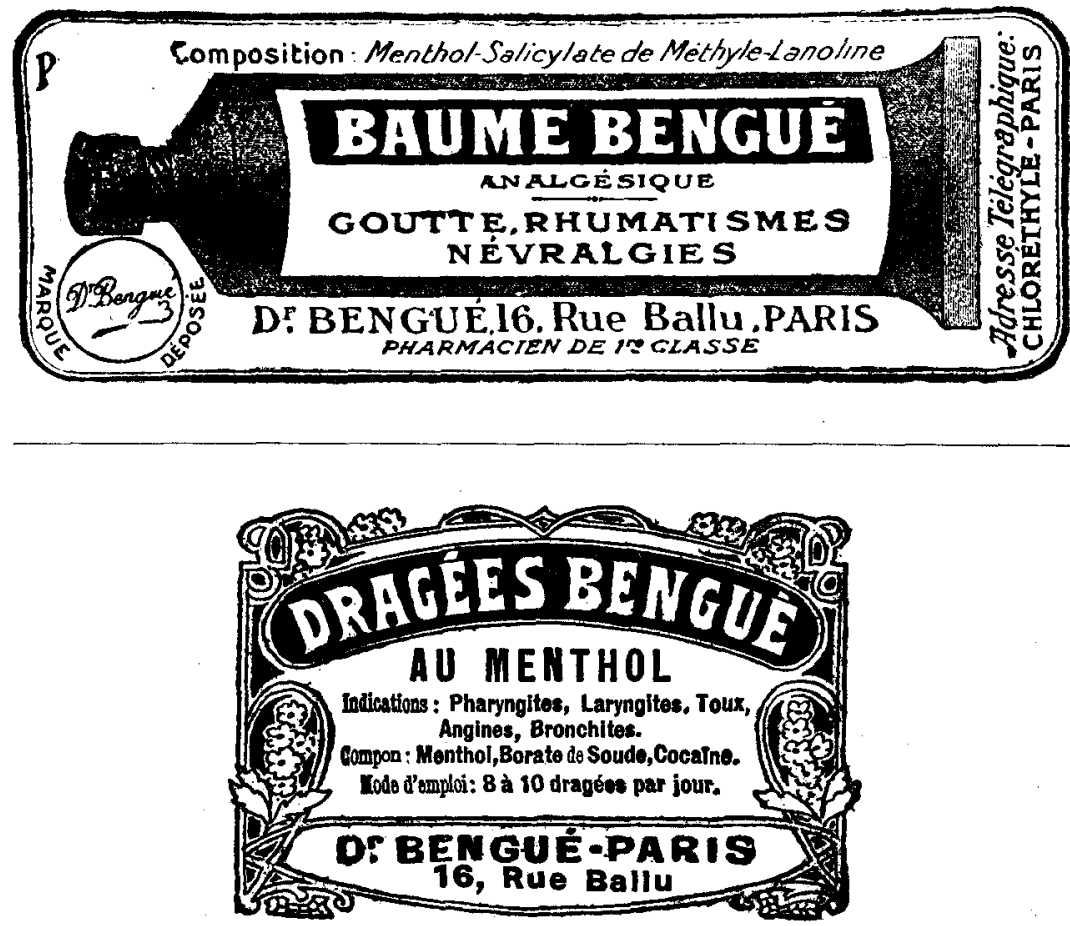
Editenrs : FÉLIX ALCAN, Paris - NICOLA ZANICHELLI, Bologne - WILLLAMS \& NOR. GATE, Londres. - AKAD. VERLAGSGESELLSCHAFT, Leipzig - G. E. STECHERT \& Co., New York. - RUIZ HERMANOS, Madrid. - RENASCENÇA PORTUGUESA, Porto - THE MARUZEN COMPANY, Tokio.

\section{"SCIENTIA"}

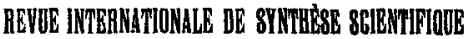

Papalssant menssellement (en lascicula de 108 a li2 yages thacas) Directeur : Eugenio RIGNANO

Est l'unique Revue collaboration vraiment internationale.

Est l'unique Revue à diffusion absolument mondiale.

Est l'unique Revue de synthèse et d'unification du savoir, qui traite les questions fondamentales de toutes les sciences : histoire des sciences, mathématiques, astronomie, géologie, physique, chimie, biologie, psychologie et sociologie.

Est l'unique Revue par conséquent qui, intéressant directement les statisticiens, les démographes, les ethnographes, les êconomistes et ceux qui cultivent le droit, l'histoire des religions et, d'une facon générale, la sociologie, par ses nombrenx et importants articles et comptes rendas relatifs à ces sciences, leur offre aussi le moyen de connâttre sous une forme résumée et synthétique, les plus grands problèmes de toutes les autres branches du savoir.

Est l'unique Revue qui puisse se vanter d'avoir parmi ses collaborateurs les savants les plus illustres du monde entier. Une liste de ceux-ci, comprenant plus de 350 noms, est reproduite dans presque tous les fascicules.

Les articles sont publiés dans la langue de leurs auteurs, et à chaque fascicule est joint un supplément contenant la traduction frangaise de tous les articles non frangais. Ainsi la revue est complètement accessible même à quí ne connaît que la langue française. Demandez un numéro spécimen gratuit au Secrétaire Général de "Scientia", Milan, en joignant à la demande, pour remboursement des frais d'envoi, la somme de quatre francs en timbres-poste de votre pays.

ABONNEMENT : Lire 200.

BUREAUX DE LA REVUE : 12, via A. de Togni - Milano (16).

Secrétaire Général de la Rédaction: D* Paolo BONETTI.

\section{BULLETIN \\ DE L'UNION DES FEMMES DE FRANCE (CROIX-ROUGE FRANÇAISE)}

\section{Bulletin mensuel}

\section{Abonnement d'un an : 5 francs}

Pour faire partie de l'Union des Femmes de France, il suffit de faire parvenir au Siège Central de I'Union des Femmes de France, 102, Boulevard Malesherbes, à Paris, une des cotisations suivantes :

de 5 à 10 francs comme Membre adhérent;

de 10 à 30 francs comme Membre titulaire.

Rachat de cotisation :

Membre bienfaiteur : 1000 francs. - Membre perpetuel : 200 francs. 


\section{Adresses des Comités centraux (suite).}

* BELGIQUE. - Croix-Rouge de Belgique, rue de Livourne, 80, Bruxelles. BOLIVIE. - Croix-Rouge bolivienne, Casilla do Correo, 504, La Paz. BRESIL. - Croix-Rouge brésilienne, Praça Vieira Souto, 10, Rio de Janeiro. BULGARIE. - Croix-Rouge bulgare, 19, Bd. Totleben, Sofia.

CANADA. - Croix-Rouge canadienne, 410, Sherboume Street, Toronto. CHILI. - Croix-Rouge chilienne, Correo, 8, Casilla 7567, Santiago de Chile. CHINE. - Croix-Rouge chinoise, Pélkin et 26, Kiukiang Road, Shanghaï. COLOMBIE. - Croix-Rouge colombienne, Bogota.

COSTA-RICA. - Croix-Rouge costaricienne, Calle 5. Sur., San José.

CUBA. - Croix-Rouge cubaine, Ignacio Agranonte et av. de Belgica, La Havane.

DANEMARK. - Croix-Rouge danoise, Ved Stranden, 2, Copenhague, $\mathrm{K}$.

DANTZIG. - Croix-Rouge de Dantzig, Neugarten, 12-16, Dantzig.

REPBLIQUE DOMINICAINE. - Croix-Rouge dominicaine, St-Domingue.

EGYPTE. - Croissant.Rouge égyptien, Ismail Pacha, $\$$ (Garden City) Le Caire.

EQUATEUR. - Croix-Rouge équatorienne, Venezuela 46, Quito.

ESPAGNE. - Croix-Rouge espagnole, Calle de Sagâsta, 10, (Estafeta, 4), Madrid.

ESTONIE. - Croix-Rouge estonienne, Niguliste Tän, 12, Reval.

ETATS-UNIS. - Croix-Rouge américaine (Central Committee of the American National

Red Cross), National Headquarters, Washington, D.C.

FINLANDE. - Croix-Rouge de Finlande, 16, Annankatu, Helsingiors.

FRANCE. - Croix-Rouge française, rue François ler, 21, Paris, $8 \mathrm{me}$.

** GRANDE-BRETAGNE. - Croix-Rouge britannique (British Red Cross Society),

Berkeley Street 19, London W. 1 .

GRECE. - Croix-Rouge hellénique, rue Solon, 39, Athènes.

GUATEMALA. - Croix-Rouge guatémalienue, 13, Calle Oriente, 2, Guatémala.

HONGRIE. - Croix Rouge hongroise, Baross utca 15, Budapest, VIII.

INDES. - Croix-Rouge des Indes (Indian Red Cross Society), New-Dehli.

ISLANDE. - Croix-Rouge islandaise, Reykjavik.

ITALIE. - Croix-Rouge, italienne, via Toscana, 12, Rome (25).

JAPON. - Croix-Rouge japonaise, 5 Shiba Park, Tolico.

LETTONIE. - Croix-Rouge lettone, Skolas ielá, 1, Riga.

LITHUANIE. - Croix-Rouge lithuanienne, Kestucio g-vé 8, Kaunas.

LUXEMBOURG. - Croix-Rouge luxembourgeoise, Luxembourg.

MEXIQUE. - Croix-Rouge mexicaine, 2, a de San Geronimo, 14, Mexico, D.F.

NORVEGE. - Croix-Rouge norvégienne, Grev Wedels Plass 5, Osla.

PANAMA. - Croix-Rouge de Panama, Panama.

PARAGUAY. - Croix-Rouge paraguayenne, Avenida Espana, 505, Assomption.

*** PAYS-BAS. - Croix-Rouge néerlandaise, Princessegracht, 27, La Haye.

PEROU. - Croix-Rouge péruvienne, Melchormalo 349, Lima.

PERSE. - Lion et Soleil-Rouges de Perse, Téhéran.

POLOGNE. - Croix-Rouge polonaise, rue Smolna, 6, Varsovie.

PORTUGAL. - Croix-Rouge portugaise, 1, Praça do Commercio, Lisbonne.

ROUMANIE. - Croix-Rouge roumaine, 40 , rue N. Balcesco, Bucarest.

SALVADOR. - Croix-Rouge de Salvador, 13 a Avenida Norte y 9 a calle Poniente, San Salvador.

ROYAUME S.C.S. - Croix-Rouge des Serbes, Croates et Slovènes, Simina ulica, 21, Belgrade. SIAM. - Croix-Rouge siamoise, Bangkok.

SUEDE. - Croix-Rouge suédoise (Svenska Roda Korset), Artillerigatan, 6, Stockholm, 14. SUISSE. - Croix-Rouge suisse, Taubenstrasse, 8, Berne.

TCH ECOSLOVAQUIE. - Croix-Rouge tchécoslovaque, Neklanova ul., 147, Prague VI. TURQUIE. - Croissant-Rouge ture, Angora.

**** U.R.S.S. (Union des républiques soviétiques socialistes). - Alliance des sociétés de la

Croix-Rouge et du Croissant-Rouge de l'U.R.S.S., Ipatievsky péréoulok, 6, Moscou.

URUGUAY. - Croix-Rouge uruguayenne, Piedad 13.779, Montevideo.

VENÉZUELA. - Croix-Rouge vénézuélienne, Muñoz a Piñango, 10, Caracas.

\footnotetext{
- Section:

Ancienne

Branche: Croix-Rouge néo-zélandaise, P.O. Box 969, Wellinglon, N.Z.

*.* Branche :

*** Membres Croix-Rouge arménienne, Erivan.

de Croissant-Rouge de l'Azerbéidjan, rue Zévine, 1, Bakou.

l'Alliance : Croix-Rouge géorgienne, 15, rue Krylov, Tiflis.

Croix-Rouge russe (R.S.F.S.R.), Pouchetchnaia, 5, Moscou,

Croix-Rouge de la Russie Blanche, 3, rue Marx, Minsk.

Croissant-Rouge du Turkménistan, Pollorutzk

Croix-Rouge ukranienne, 7 , rue du Thétre, Kharkov.

Groissant-Rouge de l'Uzbékistan, ul. Respublikanskaia, 13, Samarkand.
} 


\title{
Sommaire du Bulletin
}

\author{
T. LX, $\mathbf{N}^{\circ} 324$
}

Aour 1929

Comité international de la Croix-Rouge. - Dous pour les victimes du tremblement de terre en Perse, 717.

Institut international d'études de matériel sanitaire. - Dons, 717.

Ligue. - Vers la santé, 718.

Hongrie. - La Croix-Rouge hongroise en 1928, 718.

Indes. - Statuts de la Croix-Rouge des Indes, 720.

Italie. - Journée de la Croix-Rouge, 731.

Turquie. - Secours aux territoires atteints par la sécheresse, 731.

La Revue internationale de la Croix-Rouge, Bulletin international des Sociétés de la Croix-Rouge, parait à la fin de chaque mois.

Prix, franco, un an: Fr. 20.- Le numéro: Fr. 2.-

Années 1919, 1920 et 1921 (rares): Fr. 30.-

Administration : Promenade du Pin, 1, Genève. 